P138 (continued)

identify resources for this under served population were investigated.

Evaluation: Teacher/Caregiver observations were used as the evaluation for this initiative. Improvements were seen in both physical activity and nutrition behaviors.

Conclusions and Implications: This curriculum meets the needs of providing nutrition education to this vulnerable population. Continued outreach into this population will empower this under served population to make healthier choices and improve nutrition outcomes.

Funding: Supplemental Nutrition Assistance Program Education

\section{P139 Seniors Eating Well}

Lynn James, MS, LDN, RD, Ljames@psu.edu, Penn State Extension, 443 Plum Creek Road, Sunbury, PA 17801; Dori Campbell, MS, LDN, RD, Penn State Extension

Objective: To improve the health, nutrition knowledge and behaviors of older adults, specifically reducing sodium, increasing healthy fats and snacks, fruits, vegetables and whole grains, reading nutrition labels, and physical activity.

Theory, Prior Research, Rationale: Based on U.S. census data, 2015- 2020 U .S. Dietary Guidelines, and Social Cognitive Theory, and prior program impact research over ten years.

Description: Target audience was middle-older and older adults. Seniors Eating Well is a nine lesson curriculum that was completely updated in 2016 to incorporate the new U.S. Dietary Guidelines. It is appropriate for SNAP-Ed and other older adult audiences at senior centers, community centers, and senior housing centers and with middle-older adults (aged 5065 ) at work sites, clubs, churches, or other organizations. Lesson topics: Great Grain Discoveries, All Star Senior Snacks, Heart Healthy Meals, Cooking \& Seasoning with Herbs, Savory Soups, Fitness Fun, Evaluating Dietary Supplements, Dietary Fat Fact or Fiction, and Energy Density. Each 45 minute lesson includes lesson plans, PowerPoints, recipes, handouts and evaluations. Some lessons include games; all are discussion based.

Evaluation: Short retrospective surveys after each lesson and three-six month follow-up. Student t-tests have shown positive significant changes.

Conclusions and Implications: The educational matrix in the appendix shows how each lesson's objectives conform to the U.S. 2015 Dietary Guidelines. Over the last ten years the program has shown positive impact by increasing knowledge, skills/abilities, intent to change behaviors, and behavior change. The program has also been used by many others states' Extension services, SNAP-Ed and by older adult community programmers.

Funding: None

\section{P140 SIPsmartER: Adapting an Evidence- based Dietary Intervention Program to the Hispanic Population}

Diana Cuy Castellanos, PhD, RD, dcuycastellanos1@ udayton.edu, University of Dayton, 300 College Park, Dayton, $\mathrm{OH} 45469 ;$ Beth Miller, PhD, RD, Miami

University

Objective: Cultural adaption frameworks or processes for dietary interventions are limited. The purpose of this study was to identify and implement a best practice protocol for cultural adaptation of the SIPsmartER dietary intervention to the Hispanic population. SIPsmartER is an evidence-based dietary intervention targeting sugar-sweetened beverage behavior and was originally developed for a rural non-Hispanic white population.

Study Design, Setting, Participants: A systematic review was conducted to identify studies adapting dietary interventions to the Hispanic population. The SIPsmartER intervention was translated from English into Spanish and culturally adapted to the Hispanic population guided by frameworks and processes identified from the systematic review. Thirty-three Hispanic families, three researchers and five key informants from Southwest Ohio were involved in the adaptation process.

Outcome Measures and Analysis: Literature review, key informant observations of the curriculum delivery, target population feedback on written and visual materials, researcher experience, focus groups and mobile technology surveys were used to gain information for adaptation.

Results: During the systematic review, eight articles were reviewed and two specific cultural-adaption frameworks were identified. Using the heuristic framework and Five Categorical Strategies for Adaptation to guide the adaptation process, the SIPsmartER intervention was adapted to promote cultural-appropriateness through corrections in translation, changes of images in the educational materials, inclusion of family and peer educators, logistical considerations and the development of mobile technology.

Conclusions and Implications: The SIPsmartER intervention was culturally adapted to a Hispanic population. The adapted intervention will be tested in a larger research project to include a technological component.

Funding: University of Dayton Research Institute

\section{P141 Small Bites, Big Change! Teacher- Facilitated Nutrition Program Increases Healthy Eating Knowledge and Vegetable Consumption}

Ashley Roth, MS, LDN, RD, aroth@commonthreads.org, Common Threads, 3811 Bee Caves Road, Suite 108, Austin, TX 78746; Natalie Goldsworthy, MS, Common Threads; Stephanie Folkens, BA; Neilé Edens, PhD 